\title{
ASPECTOS MÉDICOS DE LA LITERATURA CULINARIA ÁRABE
}

\author{
Manuela MARÍN \\ C.S.I.C. Madrid
}

Las técnicas y prácticas culinarias desarrolladas en el marco de la cultura árabe-islámica medieval se conocen con cierto detalle gracias a la existencia de un legado escrito de notable extensión. Aunque de muchas obras relacionadas con la alimentación sólo se conocen los títulos ${ }^{1}$, los recetarios árabes que se han conservado componen el conjunto de textos culinarios medievales más abundante que se conoce. $\mathrm{Su}$ recuperación y estudio, iniciada por Habỉb Zayyāt y Maxime Rodinson, está aún lejos de verse completada, aunque los textos ya editados permiten la aproximación a toda una serie de cuestiones relacionadas con la alimentación en las sociedades islámicas medievales.

No.son los recetarios, por otra parte, los únicos documentos utilizables para el conocimiento de esas cuestiones; sin entrar a detallar las posibilidades que ofrecen los textos literarios o históricos, es necesario tener en cuenta la existencia de una tradición médica sobre temas de dietética e higiene alimentaria. En este trabajo voy a centrar mi atención en el estudio de las huellas de esa tradición en los libros de

1 Véase la introducción de los editores a la edición de Ibn al-Sayyār alWarrāq, Kitäb al-Tabij (ed. K. Ohmberg y S. Mroueh, Helsinki, 1987); Rodinson, M., "Recherches sur les documents arabes relatifs à la cuisine". R.E.I. (1949) 95-165, el vol. I de la edición de Al-Wusla ilà l-habtb (Alepo, 1986), con un amplio estudio de $S$. Mahŷūb y la introducción (a cargo de $\mathrm{D}$. Waines y $\mathrm{M}$. Marín) a la obra colectiva La alimentación en las sociedades islámicas (Madrid: A.E.C.I., 1994). 
cocina árabes $\mathrm{y}$, más concretamente, los que se compusieron en las regiones islámicas orientales.

Son éstos últimos los textos más numerosos y los que, en líneas generales, conceden una mayor atención a los aspectos médicos de la alimentación. El más antiguo de estos recetarios es el recopilado por Ibn al-Sayyār al-Warrāq, bajo el título de Kitäb al-Tabit $\tilde{j}^{2}$, en el s. IV/X. De época posterior son al-Wușla ila l-habib, de autor incierto ${ }^{3}$ y el Kitāb al-Tabij de al-Bagdādī; ambas obras proceden del siglo VII/XIII. Pocas precisiones cronologicas pueden hacerse, por su parte, respecto al cuarto de los recetarios de procedencia oriental. En efecto, el anónimo Kanz al-fawá 'id fítanw' al-mawá 'id carece de toda indicación interna que permita situarlo con exactitud tanto en el tiempo como en el espacio, aunque es evidente que su contenido responde a la tradición culinaria oriental. Su posible conexión con el Egipto mameluco se basa en algunas coincidencias de vocabulario y contenido con otros textos de ese periodo, pero no es, ni mucho menos, definitiva ${ }^{4}$.

La presencia de la tradición médica en estos cuatro recetarios orientales ofrece notables diferencias. Ausente por completo en alBagdādī, aparece tímidamente en la Wusla, para adquirir una mayor entidad en al-Warrāq y en Kanz al-fawã id. Examinaré a continuación las formas que esa tradición adquiere y cómo se presenta en un tipo de obras, cuyos títulos no suponen, en principio, otro contenido que el puramente culinario.

Los materiales de tipo médico y, más concretamente, dietético, aparecen en los recetarios en dos formas. En primer lugar, ciertas recetas pueden llevar indicaciones respecto a sus posibles beneficios para la salud y el bienestar de quien las consume. También en ocasiones

${ }^{2}$ Véase también "Ibn Sattār al-Warräq's Kitäb al-wusla ilà al-habib / Kitäb al-tabbakh, another MS. of Ibn Sayyār al-Warrāq's Kitab al-tabikh", de K. Ohnberg, en La alimentación en las sociedades islámicas (Madrid: A.E.C.I., 1994), p. 23-32.

3 Véase M. Rodinson, "Recherches", p. 126-130 y la introducción a la edición de la Wusla por $\mathrm{S}$. Mahŷùub, que atribuye su composición a Ibn al-"Adīm (m. 660/1262), basándose en razones paleográficas.

${ }^{4}$ Véase la introducción de los editores (M. Marín y D. Waines) al texto, publicado en Bibliotheca lslamica, 40 (Beirut, 1993). 
se hacen notar los efectos curativos que determinadas preparaciones culinarias pueden tener sobre estados de enfermedad o para corregir inclinaciones perjudiciales del organismo. Una segunda fórmula de conexión con el ámbito de la medicina se observa en los recetarios cuando éstos incluyen materiales diseñados específicamente para el tratamiento de enfermedades, ya se trate de recetas aisladas $o$, en ocasiones, de capítulos enteros dedicados a este fin. Mientras en el primer caso se suele tratar de indicaciones dispersas y escasamente sistemáticas, la introducción de recetas médico-culinarias se produce generalmente en un contexto bien definido, que corresponde a la confección de remedios y preparados curativos en un ámbito doméstico.

El más antiguo de los recetarios conservados, el de Ibn al-Sayyār al-Warrāq, es también, como ya he indicado, uno de los más ricos en este aspecto. Sus treinta primeros capítulos (la obra contiene un total de 132, de muy desigual extensión) están en su mayor parte dedicados a cuestiones dietéticas y tratan sobre la comida conveniente para jóvenes y ancianos y las cualidades generales de la alimentación (según sea dulce, ácida, astringente, picante o sosa), ampliando este tipo de información en capítulos dedicados a las facultades y perjuicios de los diferentes tipos de carne y las diversas formas de prepararla, los pescados, aves, cereales, verduras, productos lácteos, aceites, especias, perfumes y frutos, por este orden. Los capítulos 22, 24 y 25 están dedicados a las cualidades médicas de varias preparaciones culinarios: comidas "frías", encurtidos y salsas. Esta primera parte del $K$. al-Tabij de al-Warrāq se cierra con unos breves capítulos dedicados a la comida según las estaciones del año, la alimentación apropiada para el estómago enfermo, la conveniencia del ejercicio físico antes de comer y las formas de evitar los perjuicios de la alimentación.

Además de esta introducción general de carácter bromatológico, la obra de al-Warrāq contiene varios capítulos dedicados a la alimentación específica para los enfermos: $\operatorname{los} \mathrm{n}^{\mathbf{0}} 105$ (sobre las muzawwarät ${ }^{5}$ de verduras), 106 (sopas y caldos), 107 (comidas adecuadas para personas

5 Véase, sobre este tipo de platos, D. Waines y M. Marín, "Muzawwar: counterfeit fare for fasts and fevers", Der Islam, 69 (1992), 289-301. 
acatarradas), 109 (para los aquejados de ictericia). Finalmente, alWarrāq dedica dos capítulos (125 y 126) a recetas de compotas, arropes y preparados digestivos ( $\hat{y} a w a ̈ r i s ̌ n a t)$, de claro contenido farmacológico.

El análisis detallado de todos estos materiales de tipo médico en la obra de al-Warrāq excede los límites de este trabajo. Para dar, con todo, un ejemplo de su interés por estos temas y del tratamiento que de ellos ofrece, he seleccionado en el $K$. al-Tab $j_{j}$ los datos relativos a las bebidas, que presento a continuación ${ }^{6}$.

No existe, en este recetario, un capítulo especial dedicado a líquidos medicinales. Sin embargo, los que se ocupan de las bebidas en general ( $\mathrm{n}^{0} 112$ a 124) contienen diversas indicaciones en este sentido; uno de ellos ( ${ }^{\circ}$ 112), muy breve, se refiere a los beneficios y perjuicios producidos por el fuqqa ${ }^{7}$, otro $\left(\mathrm{n}^{0} 118\right)^{8}$ se compone de tres recetas de bebidas calmantes de la calentura y un tercero $\left(\mathrm{n}^{\circ} 115\right)$ hace recomendaciones sobre las bebidas más adecuadas a cada temperamento. Hay otras recetas con indicaciones médicas: una bebida especialmente indicada para el fró en los riñones y el estómago y para el dolor de cadera que, además, estimula el apetito y purifica el estómago ${ }^{9}$. En un capitulo dedicado a bebidas confeccionadas a base de distintas verduras $\left(n^{\circ} 122\right)$ se recomienda una de alcaparras para los gases intestinales, dos más, una de zanahorias y otra con garbanzos, para reconfortar los riñones y el estómago y aumentar la potencia sexual y por último, una bebida de amapolas que remedia el dolor de pecho y la tos. Entre las bebidas a base de frutas frescas, la de agraz se recomienda para la calentura y la bilis ${ }^{10}$. Por último, una de las tres recetas de mayba $a^{11}$

${ }^{6}$ Traduzo de forma general ašriba por "bebidas", aunque en casos concretos los textos se refieran a compuestos de la consistencia de los jarabes.

${ }^{7}$ Sobre este tipo de bebidas, cf. F. Aubaile-Sallenave, "Parfums, épices et condiments dans l'alimentation arabe médiévale", en La alimentación en las sociedades islámicas, p. 233 y ss.

${ }^{8}$ Los editores han respetado el error del ms. original, que da a este capítulo el $n^{\circ} 128$.

${ }^{9}$ K. al-Tabï, p. 302.

${ }^{10}$ Idem, p. 315.

11 Aunque mayba se define usualmente como una bebida a base de zumo de membrillo (véase Ibn Rasūl, Al-Mu'tamad fil-adwiya al-mufrada (Beirut, 1975), 
que contiene el capítulo $124^{12}$ está indicada para los estados de debilidad, yá que fortifica el estómago.

Parece evidente que al-Warrāq está siguiendo, en el caso de las bebidas, el primero de los esquemas a que hacía alusión más arriba; es decir, el material médico no constituye un bloque homogéneo, como ocurre con los primeros capítulos de su obra, sino que se intercala de forma más o menos continuada, dentro de una serie de recetas en las que muchas no tienen indicaciones curativas. El caso de las bebidas, con todo, no puede aplicarse al resto de la obra, en el que las recomendaciones terapéuticas son mucho menos abundantes. Esto es así porque las bebidas forman en si mismas un capítulo especial de la literatura farmacológica, que sin embargo no suele ocuparse de las confecciones culinarias. El trasvase de esta información de tipo médico -procedente de textos especializados- hacia los recetarios de cocina sigue un camino paralelo al de la inclusión, en estos recetarios, de fórmulas para la preparación de digestivos, arropes y compotas medicinales. Si estos últimos materiales tienen únicamente una función curativa, en el caso de las bebidas se aprecia mejor la fusión de dos tradiciones, la médica y la culinaria. De esta forma, entre los capítulos dedicados a ašriba, alWarrāq incluye tanto los ya citados como otros que se ocupan de la preparación de bebidas a base de productos lácteos, frutas y verduras, miel y azúcar, etc.; se trata de un conjunto de recetas en las que no aparece ningún comentario o indicación de tipo médico, pero que se encuentran intercaladas, a veces en el mismo capítulo, con las señaladas anteriormente.

Este sistema de incorporación de las informaciones médicas es el mismo que practica el autor de la Wuṣla ild l-habib, aunque en una proporción mucho menor que la empleada por al-Warrāq. Carece esta obra, además, de capítulos especialmente dedicados a la dietética; todo ello hace que pueda pensarse en una disminución, tanto cuantitativa como cualitativa, de la importancia de la tradición médica en la literatura culinaria oriental. A ello se añade que el texto de al-Bagdādī,

p. 511; Ibn al-Haššā', Glossaire sur le Mans'uri de Razès, ed. G.S. Colin y H.P.J. Renaud (Rabat, 1941), $\mathrm{n}^{\circ} 715$ ), en este caso se confecciona a base de zumo de manzanas ácidas.

${ }^{12}$ P. 316. 
que tiene una relación muy estrecha con la Wuṣla, prescinde por completo de este tipo de materiales.

Las recomendaciones médicas en la Wușla aparecen principalmente en dos capítulos no estrictamente culinarios: el $1^{\circ}$, sobre perfumes y el $2^{0}$, sobre bebidas. Una única receta de carácter culinario contiene indicaciones en este sentido: un plato llamado sahn $\bar{a}^{t}$ kaddăba ${ }^{13}$, que se recomienda para los que padecen de una complexión biliosa y para reconfortar el estómago. En cuanto al capítulo sobre perfumes, la Wuṣla ofrece una receta de polvos de olor (dartra) ${ }^{14}$ para "reforzar" el cerebro y el frio de la cabeza y otra de un óleo $(d u h n)^{15}$ que contrarresta el dolor de pecho, los gases fríos y con flema, y el frío de la cabeza.

De mayor interés para el tema que nos ocupa aquí es el capítulo $2^{\circ}$, sobre las bebidas, capítulo que ofrece además un punto de referencia y comparación con lo ya visto en al-Warrāq. Como en el resto de la obra, el autor de la Wuṣla es mucho más restrictivo que al-Warrāq a la hora de incorporar materiales médicos. En este apartado de las bebidas, únicamente cuatro recetas aportan esta clase de indicaciones: una de habb rummān ${ }^{16}$, que es buena para las náuseas y la digestión pero debilita el coito y el estómago; otra de sukkar wa-laymün ${ }^{17}$, de la que sólo se afirma que es una bebida más beneficiosa que el fuqqa', sin entrar en más detalles, y finalmente, un electuario (la' $\bar{u} q)$ contra las náuseas y el vómito y un dawă qaraf ${ }^{8}$.

13 Wusla, p. 690. El nombre de la receta (sahna) debería indicar su confección a base de pescado. El calificativo de "engañosa" se explica porque en su preparación no se utilizan más que verduras y especias. Otros casos de platos "engañosos" pueđen verse en al-Warrāq, p. 121 y ss., donde también aparece una șahna $\bar{a}^{t}$ sin pescado (mucho más sencilla que la de la Wuṣla) o una tortilla sin huevos.

$$
\begin{aligned}
& { }^{14} \text { P. } 489-90 . \\
& { }^{15} \text { P. } 497 . \\
& { }^{16} \text { P. } 506 . \\
& { }^{17} \text { P. } 507 . \\
& { }^{18} \text { P. } 508-509 .
\end{aligned}
$$


La presencia de estos dos últimos términos y sus correspondientes recetas merece algún comentario. Suponen ambas, en primer lugar, una innovación con respecto a al-Warrāq, que no las utiliza. Los electuarios pertenecen a la tradición puramente farmacológica: son remedios per se, no preparaciones como las señaladas por al-Warrāq, que tienen un doble carácter culinario y terapéutico. Como tales, aparecen regularmente tanto en las obras dedicadas a los medicamentos compuestos como en los capítulos a ellos dedicados en los tratados médicos generales ${ }^{19}$. La aparición en la Wuṣla de una receta de este tipo podría responder a la incorporación directa de preparados médicos a la literatura culinaria.

Por su parte, la receta de dawä' qaraf no incluye indicaciones terapéuticas, pero su propio nombre es significativo: se trataría de un tónico contra la desgana y la falta de apetito. No he podido encontrar, en los tratados farmacológicos que he consultado, referencia alguna a este preparado, quizá porque no se considerase como una medicina. Volveremos a encontrar referencias a este tipo de confecciones al tratar del tercero y último de los textos culinarios que estamos examinando.

En conjunto y tal como se ha indicado anteriormente, los aspectos médicos de la Wuṣla son mucho más reducidos y esporádicos que los contenidos en la obra de al-Warrāq. Su autor prescinde por completo de orientaciones dietéticas generales y concentra su mayor interés por estos asuntos en dos capítulos que no tienen que ver, en sentido estricto, con la preparación de alimentos. Sin embargo, la presencia de terminología médica e incluso de recetas -aunque en número muy escaso- de carácter terapéutico permiten asegurar la continuidad de una orientación definida a este respecto, que se verá acentuada en la obra que examinaré a continuación.

19 Véase Levey, M., The Medical Formulary or Aqräbāahin of al-Kindi (Madison, 1966), no 110, 111, 112, 114, 115; Sahlān b. Kaysān, en P. Sbath y C.D. Avierinos, Deux traités médicaux (Cairo, 1953), p. 59-60 del texto árabe; Ibn Sinā, Al-Qänün fĭ l-tibb (ed. I. al-Qašš y 'A. Zay'ūr, Beirut, 1987), V, p. 2345-47; Al-Qalānisī, Aq̣răbādín (ed. M. Z. al-Bābā, Alepo, 1983), p. 154 (cfr. Fellmann, I., Das Aqräbădin ăl-Qalanisi (Beirut, 1986), p. 223); Valverde, J.L. y Peña Muñoz, C., El formulario de los hospitales de lbn Abīl-Bayān (Granada, 1981), capitulo 5; M. Levey y N. al-Khaledy, The Medical Formulary of AlSamarqandi (Philadelphia, 1967), p. 154. 
Los materiales médicos en el Kanz al-fawa'id se presentan siguiendo un esquema parecido al de al-Warrāq: tres capítulos completos de este carácter ${ }^{20}$, junto a indicaciones dispersas a lo largo de toda la obra. A diferencia de al-Warrāq, el Kanz no incluye capítulos sobre las características de los alimentos y sus beneficios o perjuicios, aunque recoge información de este tipo referida únicamente a productos lácteos y encurtidos ${ }^{21}$. En toda la obra se encuentran, también como en alWarrāq, y nunca de forma sistemática, diversas acotaciones sobre los efectos de determinados platos en la salud de las personas.

Los tres capítulos que muestran una mayor relación con la medicina son el $8^{\circ}$ : muzawwarät de verduras para alimentación de los enfermos; el $11^{\circ}$ : electuarios y bebidas para tomar antes y después de las comidas, y el $14^{\circ}$ : confección de adwiyat al-qaraf.

El capítulo octavo consta de 23 recetas, lo que representa un notable incremento sobre las diez de este tipo que recoge al-Warrāq. En muchos casos se especifica para qué tipo de enfermos son especialmente beneficiosas: así, las muzawwarät para quien padece de fiebre $\left(\mathrm{n}^{0} 205\right.$, $207,224)$, de bilis $\left(n^{\circ} 206,224\right)$, de desgana $\left(n^{0} 209\right)$ o de miedo y opresión ( $\left.\mathrm{n}^{\circ} 210\right)$. Otras dos recetas ( $\mathrm{n}^{0} 226$ y 227 ) cubren un espectro más amplio, ya que la primera es beneficiosa para los que padecen de complexión biliosa, inflamación en el hígado, debilidad de estómago, ictericia, oclusión en el hígado y el bazo e hidropesía, mientras que la segunda conviene para la fiebre biliosa y la inflamación de hígado, calma la sed, corta las náuseas y es apropiada para los cuerpos enfermos. Un dato de interés es que en una de estas recetas de muzawwara $\left(\mathrm{n}^{\circ} 207\right)$ se recomienda el servirla al enfermo en un cuenco (mi'laqa).

En el capítulo 11 se encuentran 44 recetas de digestivos, electuarios

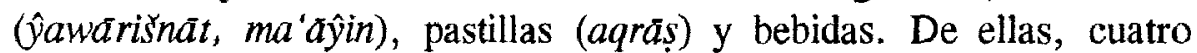

20 Véase un resumen del contenido de Kanz en Marín, M., "Las plantas alimenticias y su utilización en un manuscrito oriental sobre alimentación y dietética", Ciencias de la Naturaleza en al-Andalus, ed. E. García Sánchez (Granada, 1990), 207-222.

${ }^{21} \mathrm{Kanz}, \mathrm{n}^{\circ} 603$. 
están dedicadas a la preparación del oximiel ${ }^{22}$, a lo que hay que añadir diferentes tipos de bebidas: moyba $\left(\mathrm{n}^{\circ} 350\right)$, mähida $\left(\mathrm{n}^{\circ} 351\right)$ y un buen número de variedades de $\$ a r a b\left(n^{0} 352,353,357,360,361,362,365\right.$, 366,367 y 368). Las recomendaciones médicas de este capitulo se concentran en el oximiel y en varios tipos de yawarišnat $\left(\mathrm{n}^{\mathbf{0}} 347-49\right.$ y 372-77); sorprende en cierto modo no encontrar la misma clase de información en el resto de las recetas, ya que todas ellas proceden de la tradición farmacológica y por esta misma razón se encuentran agrupadas en un solo capítulo. Todo este conjunto de recetas parecen, por otra parte, carecer de una ordenación sistemática; se diría que el autor ha coleccionado, de fuentes diversas, una serie de materiales con el común denominador de proceder de recetarios farmacológicos, limitándose a reproducirlos tal como le venían a las manos. Este modo de proceder puede apreciarse igualmente en los capítulos exclusivamente culinarios de la obra. Está claro, con todo, que el Kanz diferencia entre este tipo de bebidas (aśriba) y las que aparecen en el capítulo siguiente (n n $^{0}$ 12: fuqqā', aqsima', sašs, $m \tilde{a}^{\prime}$ al-ša 'tr, sübiya), y en el que sólo hay una recomendación de carácter médico, referida a una receta de fuqqa' ( $n^{\circ} 397$ ) que calma la sed y es buena para los biliosos y los que padecen estados febriles.

Si la Wușla contenía una preparación de dawa' al-qaraf, el Kanz dedica a estas preparaciones todo un capitulo, de cuyas 26 recetas sólo seis no corresponden a esta denominación y se aplican a otras confecciones (dos recetas de sals $\left(\mathrm{n}^{0} 442 \text { y } 443\right)^{23}$, dos de habb rummän $\left(\mathrm{n}^{\circ}\right.$ 450 y 452), y una de la úq $\left(n^{0} 449\right)$, de sukkar wa-laymün $\left(n^{0} 451\right)$ y de närany $\left(\mathrm{n}^{\circ} 453\right)$. En varias de estas recetas se indica que son útiles para combatir las náuseas y el vómito, pero sólo en una de las adwiyat al-qaraf $\left(n^{0} 461\right)$ se hace notar que es beneficiosa para la cefalea y la fiebre.

Se trata por tanto de un capítulo de clara inclinación terapéutica. $\mathrm{La}$ falta de referencias a este respecto en la mayoría de las recetas no es

22. Véase Waines, D. y Marín, M., "Ibn Sinā on sakanjabīn" (en curso de publicación).

${ }^{23}$ Vease M. Rodinson, "Recherches", p. 142 y nota 6, donde afirma que esta palabra sólo está documentada en Wuṣla y sugiere para ella un origen romance. Kanz añade por tanto un nuevo testimonio para la utilización de este término. 
significativa, ya que su propio nombre es revelador de la finalidad a que están destinadas. La composición de estas "medicinas" es muy variada y su consistencia tampoco es uniforme, ya que en algunos casos de trata de líquidos espesos y en otros de preparados sólidos. Los elementos que aparecen con mayor frecuencia en estas recetas son el azúcar, vinagre, zumos aromáticos, frutas, especias e hierbas. Como ejemplo, traduzo a continuación una de estas recetas, de dos que aparecen calificadas como las mejores y de mayor bondad y que tienen un contenido muy semejante:

\section{$\mathrm{N}^{\circ} 446$}

"Receta de dawã' qaraf de maravillosa excelencia y bondad.

Se cogen dos onzas (üqrya) de granos de granada $\hat{y} u ̈ r^{24}$ y se machacan muy bien en un mortero de piedra junto con hojas de hierbabuena lavadas, hasta que se mezcla todo. Se le vierten dos onzas de agua de rosas del país, se le exprimen dos limones verdes y se le añade a todo ello media onza de azúcar. Se pone a fuego lento y se remueve hasta que queda todo bien mezclado. Se le espolvorea áloe $q \bar{a} q u l l l^{25}$ un poco triturado y se remueve con un palo de sauce o una rama de hierbabuena hasta que todas sus partes quedan fundidas. Se le pone un poco de agua de rosas azmiclada y se retira".

Otra receta puede servir para ilustrar las muy variadas combinaciones de ingredientes que entran en estos preparados contra la desgana y la falta de apetito:

\section{$\mathrm{N}^{\circ} 457$}

"Receta de dawā' qaraf.

Se coge agua de limón, agua de granadas ácidas, agua de rosas del país, agua de agraz, tamarindo macerado y filtrado con vinagre de vino y granos de granada machacados. Se destila todo ello, se le pone azúcar y se pone al fuego, removiéndolo con dos ramas de hierbabuena. Se le

${ }^{24}$ Este adjetivo, correspondiente a la ciudad iraní de Ŷūr (luego Fìrūzābād̆; cf. Yäqüt, Mu'ŷam al-buldān (Beirut, s.d.), s.v. Yùrr) suele aplicarse a las afamadas rosas cultivadas en esta región (R. Dozy, Supplément, s.v.).

${ }^{25}$ M. Meyerhof, en sus notas al texto de Maimónides Šarh asmã' al- 'uqqăr ( $\mathrm{n}^{\circ} 296$ ), afirma que 'üd qãqulli' es el nombre usual que dan los drogueros de E1 Cairo al áloe. 
pone un pedazo de membrillo y atrăf al-țib ${ }^{26}$. Se le hacen a un limón verde cuatro hendiduras y se rellena con pimienta y aträf al-țib, echándolo a continuación en el jarabe anterior. Se deja hacer hasta que adquiere la consistencia del la'uqq. Esto lo emplean los desganados y es una de las mejores medicinas contra la inapetencia. Cada vez que la hierbabuena se marchite, se usa otra fresca".

A través de este repaso de las obras culinarias orientales ha podido observarse cómo determinados aspectos de la tradición médica fueron asimilados e incorporados por estos textos. El que obras culinarias se ocupen de temas dietéticos no tiene, en principio, nada de extraño; pero, como ya hemos visto, los tratados culinarios no se limitan a este aspecto $y$, en mayor o menor medida, proporcionan a sus lectores informaciones de tipo claramente terapéutico. Es de sobra conocido el interés de la medicina árabe por la alimentación y sus capacidades curativas y preventivas ${ }^{27}$, bien reflejado en los capítulos introductorios del $K$. al-Ṭabij de al-Warrāq. Sin embargo, lo que más llama la atención en estos tratados es la introducción de recetas destinadas a la curación de enfermedades o a la corrección de estados o situaciones perjudiciales para la salud.

Si se comparan este tipo de recetas con las contenidas en los tratados de medicamentos compuestos se observa de inmediato que los autores de libros de cocina procedieron a una selección cualitativa del material de que disponían. Los tratados farmacológicos presentan una gran variedad de confecciones médicas: digestivos, píldoras, decocciones, pastillas, polvos, jarabes, mermeladas, arropes, electuarios, gargarismos, colirios, lavativas, unturas, ungüentos, etc. ${ }^{28}$. La mayor parte de estos remedios no aparecen en los textos culinarios que, como ya se ha indicado, se limitan a algunas clases de electuarios y bebidas. Es decir, parece darse una orientación definida hacia preparaciones de

${ }^{26}$ Sobre este término, que se aplica a una combinación de diferentes especias, cf. M. Rodinson, "Recherches", p. 132.

${ }^{27}$ Cfr. M. Ullmann, Die Medizin im Islam (Leiden, 1970), p. 199-203 y S. Mahŷūūb, introducción a la edición de la Wuṣla, p. 137 y ss.

${ }^{28}$ Véase el índice de los formularios de Sahlān b. Kaysān o de Ibn Abī 1 Bayān citados en nota 18 . 
carácter médico-culinario, preparaciones de fácil confección según las técnicas culinarias y cuyos ingredientes son también de uso corriente en la preparación de los alimentos para su consumo.

Por tanto, estos tratados de cocina representan una incorporación de elementos médicos dentro de una tradición de actividades domésti$\operatorname{cas}^{29}$. No quiere ello decir que se trate de prácticas generales y extendidas a toda la sociedad; hay que tener en cuenta que la existencia de estos textos culinarios refleja las necesidades y gustos de un fragmento muy definido de esa sociedad. Se trata sobre todo de grupos sociales acomodados, que viven en un entorno urbano y tienen acceso tanto a bienes materiales muy diversos como a una serie de intercambios culturales especializados. En ese ambiente, el interés por los temas gastronómicos no se puede desligar del gusto por la poesía o por las ciencias, ni de la preocupación por el cuidado del cuerpo, como demuestran aquellos tratados de cocina que contienen capítulos especiales sobre perfumes, dentifricos y desodorantes ${ }^{30}$. La tradición médica recogida en estas obras no es más que una pequeña parte dentro del conjunto de informaciones que nos ofrecen; la gran mayoria de las recetas de cocina no contienen indicaciones dietéticas ni terapéuticas. Cuantitativamente limitada, su presencia refleja, por otra parte, la penetración de unos saberes puramente científicos en el ámbito de la vida cotidiana. Ese es, a mi modo de ver, el interés mayor que ofrece su estudio ${ }^{31}$.

29 Véase también, sobre este tema, la comunicación presentada por $D$. Waines al Congreso de la Unión Europea de Arabistas e Islamólogos (Salamanca, 1992), bajo el título "Medicinal nutrients as home remedies: a case of convergence in the medieval Middle Eastern culinary and medical tradition".

${ }^{30}$ Véanse los capítulos 20,21 y 22 de Kanz al-fawa 'id.

31 Una primera versión de este trabajo se leyó en el Congreso de Historia de las Ciencias Árabes celebrado en Granada en 1992. 